\title{
Serological and Molecular Characteristics of Pathogenic Leptospira circulating in rat populations in Fujian Province, China, 2018-2020
}

\author{
Guoying Xu \\ Fujian Center for Disease Control and Prevention \\ Haiyan Qiu \\ National Institute for Communicable Disease Control and Prevention, Chinese Center for Disease Control and Prevention \\ Weijun Liu \\ Fujian Center for Disease Control and Prevention \\ Xiugao Jiang \\ National Institute for Communicable Disease Control and Prevention, Chinese Center for Disease Control and Prevention \\ Yung-Fu Chang \\ Cornell University \\ Jiaxiong Wang \\ Fujian Center for Disease Control and Prevention \\ Zhenpeng Li \\ National Institute for Communicable Disease Control and Prevention, Chinese Center for Disease Control and Prevention \\ Yongzhang Zhu \\ Shanghai Jiao Tong University \\ Cuicai Zhang ( $\sim$ zhangcuicai@icdc.cn) \\ National Institute for Communicable Disease Control and Prevention, Chinese Center for Disease Control and Prevention \\ Fangzhen Xiao \\ Fujian Center for Disease Control and Prevention
}

\section{Research Article}

Keywords: Leptospira, Rodent, Genetic diversity, Serological characteristics

Posted Date: January 26th, 2022

DOI: https://doi.org/10.21203/rs.3.rs-1291430/v1

License: (c) (1) This work is licensed under a Creative Commons Attribution 4.0 International License. Read Full License 


\section{Abstract \\ Background}

Leptospirosis is a significant emerging infectious disease worldwide. Rodents are considered to be the most critical hosts for Leptospira spp. Fujian Province is a region highly endemic for leptospirosis in China. However, the genetic diversity of leptospires circulating among rodents in Fujian is limited.

\section{Results}

The carrier status of Leptospira spp. in rats was investigated by culturing and serological detection in Fujian during 2018-2020. A total of 710 rodents, including 11 species, were trapped, with $R$. rattoides being the dominant species (50.56\%). Fourteen pathogenic Leptospira strains were obtained. Two species including $L$. interrogans and L. borgpetersenii were identified using $16 S$ rRNA gene. Four serogroups were identified by the microscopic agglutination test (MAT), including 7 Javanica, 4 Icterohaemorrhagiae, 2 Bataviae and 1 Pyrogenes. Six STs were identified by MLST, including 7 ST143, 2 ST1, 2 ST17, 1 ST96, 1 ST332 and 1 ST333. L. borgpetersenii serogroup Javanica belonging to ST143 was the dominant type (50.00\%). A total of 387 rat serum samples were tested by MAT. Sera were considered positive for seroreactivity at a titer $\geq 1: 20$ against at least one serovar. A total of 138 ( $35.66 \%$ ) sera samples tested positive, and seven serogroups were identified, with Javanica being the dominant serogroup (81.16\%), the similar serogroup isolated from rodents. This study demonstrates a high prevalence of leptospirosis in rats and public health education among high-risk workers is highly recommended.

\section{Conclusions}

This study confirmed $R$. rattoides were the most abundantly rats, and L. borgpetersenii serogroup Javanica ST143, L. interrogans serogroup Icterohaemorrhagiae ST1 and ST17 were widely distributed among rodents in Fujian during 2018-2020. Despite the low number of isolates obtained from rodents, this study suggests that continuous epidemiological surveillance on the etiological characteristics of pathogenic Leptospira in wild animal reservoirs may help reduce the possible risk of disease transmission.

\section{Background}

As one of the most widespread zoonotic diseases, leptospirosis is caused by pathogenic spirochetes of Leptospira genus with estimated one million human leptospirosis cases and 58,900 deaths annually (1-3). The genus Leptospira is classified into pathogenic, intermediate, and saprophytic species (4, 5). Based on antigenic similarity, more than 300 pathogenic serovars are clustered into 24 serogroups. Approximately half of the reported pathogenic serogroups/serovars belong to L. interrogans or L. borgpetersenii (6).

Humans and animals can be infected primarily through direct or indirect exposure to urine or tissues of infected mammalian hosts and urine-contaminated soil or water (7). Among wildlife species, rodents are considered the most critical reservoir of pathogenic Leptospira worldwide due to their close contact with humans and infect livestock and companion animals $(1,8)$. Therefore, long-term active surveillance and investigations on the carriage status of rats will contribute to understanding animal-to-human transmission, outbreak investigation, and source tracking for leptospirosis. Although leptospirosis has significantly decreased over the past few years, leptospirosis is still recognized as a critical zoonosis in China. The disease incidence was between 0.0381 and 0.0639 cases per 100,000 during the $2018-2020$ period in Fujian Province, slightly higher than the national incidence ( between 0.0113 and 0.0212 cases per 100,000 during 2018-2020), showing that Fujian Province was a significant epidemic region for leptospirosis (data available from the National Notifiable Infectious Disease Surveillance System in China). The average incidence rates in some regions in Fujian Province, especially such as Pucheng(0.334 cases per 100,000), Luoyuan $(0.778$ cases per 100,000$)$, and Wuping $(0.361$ cases per 100,000$)$ city in Fujian, are markedly higher than the national incidence in China $(0.0159$ cases per 100,000$)$. Our previous study has reported two predominant pathogenic Leptospira named L. interrogans serogroup Icterohaemorrhagiae and L. borgpetersenii serogroup Javanica, with ST1 and ST143 being the dominant STs circulating in the rat population in Jiangxi Province in China (9). However, there is little information regarding the most crucial rat hosts and their transmission to humans in the highly endemic district of Fujian Province. Therefore, the purpose of this study was to investigate the prevalence of pathogenic Leptospira in potential reservoir animals of rodents and to identify the predominant species/serogroups/genotypes circulating in rat populations in Fujian Province. The detailed molecular and serological characteristics of Leptospira circulating in this specific area will provide deeper insights into epidemiology and prevent and control of leptospirosis.

\section{Results}

\section{Rodent distribution and isolation from Fujian Province}

A total of 9,136 traps were placed, and 710 rodents belonging to 11 different species were successfully captured between 2018 and 2020 in Fujian Province. Species identification of trapped rodents and the numbers of rodents with positive cultures were listed in Table 1. The density of rodents was between 4.81$16.77 \%$ in the six trapping sites in Fujian Province, with Luoyuan being the highest density of rodents (16.77\%). The most abundantly trapped species was $R$. rattoides $(50.56 \%, 359 / 710)$, followed by $N$. fulvescens $(19.86 \%, 141 / 710)$ and $R$. flavipectus $(18.17 \%, 129 / 710)$ in Fujian. 
Table 1

Q Rodent distribution and numbers of rodents with positive renal cultures during 2018-2020 in Fujian Province.

\begin{tabular}{|c|c|c|c|c|c|c|c|c|c|c|c|c|}
\hline regions & A & B & $\begin{array}{l}\text { Rodent } \\
\text { density }\end{array}$ & $\begin{array}{l}\text { Rattus } \\
\text { rattoides }\end{array}$ & $\begin{array}{l}\text { Rattus } \\
\text { flavipectus }\end{array}$ & $\begin{array}{l}\text { Rattus } \\
\text { norvegicus }\end{array}$ & $\begin{array}{l}\text { Niviventer } \\
\text { fulvescens }\end{array}$ & $\begin{array}{l}\text { Berylmys } \\
\text { bowersi }\end{array}$ & $\begin{array}{l}\text { Niviventer } \\
\text { niviventer }\end{array}$ & $\begin{array}{l}\text { house } \\
\text { mouse }\end{array}$ & $\begin{array}{l}\text { Apodemus } \\
\text { agrarius }\end{array}$ & $\begin{array}{l}\text { Niviventer } \\
\text { coninga }\end{array}$ \\
\hline Changtai & 1777 & 100 & 5.63 & $0 / 34$ & $2 / 55$ & $0 / 9$ & $0 / 1$ & $0 / 0$ & $0 / 0$ & $0 / 1$ & $0 / 0$ & $0 / 0$ \\
\hline Fuqing & 561 & 27 & 4.81 & $1 / 24$ & $0 / 0$ & $0 / 0$ & $0 / 2$ & $0 / 0$ & $0 / 1$ & $0 / 0$ & $0 / 0$ & $0 / 0$ \\
\hline Luoyuan & 483 & 81 & 16.77 & $1 / 76$ & $0 / 1$ & $0 / 0$ & $0 / 0$ & $0 / 0$ & $0 / 2$ & $0 / 0$ & $0 / 0$ & $0 / 0$ \\
\hline Jianyang & 378 & 37 & 9.79 & $0 / 6$ & $0 / 0$ & $0 / 0$ & $1 / 25$ & $0 / 2$ & $0 / 1$ & $0 / 0$ & $0 / 0$ & $1 / 1$ \\
\hline Pucheng & 315 & 37 & 11.75 & $0 / 2$ & $0 / 0$ & $0 / 0$ & $0 / 9$ & $0 / 0$ & $0 / 7$ & $0 / 0$ & $2 / 11$ & $0 / 2$ \\
\hline Wuping & 5622 & 428 & 7.61 & $3 / 217$ & $3 / 73$ & $0 / 11$ & $0 / 104$ & $0 / 23$ & $0 / 0$ & $0 / 0$ & $0 / 0$ & $0 / 0$ \\
\hline Total & 9136 & 710 & 7.77 & $5 / 359$ & $5 / 129$ & $0 / 20$ & $1 / 141$ & $0 / 25$ & $0 / 11$ & $0 / 1$ & $2 / 11$ & $1 / 3$ \\
\hline
\end{tabular}

A-Number of total traps placed for each region.

B-Number of rodents trapped for each region.

Rodent density calculated as (B/A * 100).

Fisher's exact test revealed highly significant differences in the species distribution of rodents across different collected sites (P $<0.001)(\mathrm{S} 3 \mathrm{Table})$. Those species represented most of the rodent diversity in Fujian Province. $R$. rattoides was the most common species in Fuqing (24/27), Luoyuan (76/81), and Wuping (217/428), R. flavipectus in Changtai (55/100), N. fulvescens in Jianyang (25/37), and A. agrarius in Pucheng (11/37).

A total of 14 strains from 5 different species of field rodent were isolated (Table 1 and Table S1). The carriage rates of these 5 different species of rodents based on culture isolation were between $0.71-33.33 \%$, with the highest rates in $N$. coning $(1 / 3,33.33 \%)$, A. agrarius (2/11, 18.18\%) and $R$. flavipectus (5/129, $3.88 \%$, indicating that these three species may be the primary carrier of Leptospira in Fujian.

Results of species identification of Fujian isolates based on 16S rRNA gene

Among the 14 isolates, species identification revealed that L. interrogans and L. borgpetersenii occupied equal to each other, respectively (Fig. 1 and Table S1). Fisher's exact test revealed highly significant differences in the distribution of leptospiral species prevalence across collected sites among the 14 strains $(p<0.001)$ (Table S4), but no differences across rodent species (Table S5). There were some special geographic differences between the dominant pathogenic Leptospira species. L. interrogans was widely represented in Jianyang, Pucheng, Fuqing and Changtai, while L. borgpetersenii was only distributed in the remaining two cities of Luoyuan and Wuping (Table S4). Furthermore, L. interrogans and L. borgpetersenii were both present every year between 2018 and 2020 (Table S1).

\section{Serogroup identification of 14 isolates from Fujian Province from 2018 to 2020}

A total of four serogroups were identified among these 14 isolates. Serogroup Javanica and Icterohaemorrhagiae, as the most prevalent serogroup, accounted for $50.00 \%$ and $28.57 \%$, respectively, followed by Bataviae (14.29\%) and Pyrogenes (7.14\%) (S1 Table).

There were some significant regional differences across six collected sites in the distribution of the dominant serogroups tested by Fisher's exact test ( $p<0.05$ ) (Table S6). Icterohaemorrhagiae was the only predominant serogroup in Jianyang and Pucheng, Bataviae in Fuqing, and Javanica in Luoyuan and Wuping, but serogroup Bataviae and Pyrogenes were identified in Changtai. In addition, serogroup Javanica was present every year between 2018 and 2020 in Wuping (Table S1).

\section{Genetic diversity of 14 Fujian Province isolates using MLST analysis}

A total of 6 different STs, including 2 new STs (ST332 and ST333) were identified from 14 pathogenic Leptospira isolates in Fujian Province (Table S1). The most predominant ST was ST143 (7/14), followed by ST1 (2/14) and ST17 (2/14). The remaining 3 isolates belonged to 3 different STs (Table S1). The UPGMA dendrogram of STs showed that the strains isolated from the same trapping sites clustered closely together except the two strains belonging to two different STs from Changtai (Fig. 2). The dendrogram revealed that the 14 pathogenic Leptospira strains belonged to five major clades. Fisher's exact test revealed highly significant differences in the distribution of ST prevalence across collected sites among the 14 strains ( $p<0.001)$ (Table S7). In addition, there were some significant regional variations in the distribution of the dominant STs. ST143 was the only typical ST in Luoyuan and Wuping, ST17 in Jianyang, ST1 in Pucheng, ST96 in Fuqing, while ST332 and ST333 were identified in Changtai (Fig. 2).

\section{MAT on Rats Sera}

Three hundred twenty-three rats had insufficient serum, and therefore a total of 387 rat serum samples from the trapped rats were tested for Leptospira antibodies by MAT with a panel of 10 local serovars. Sera were considered positive for sero-reactivity at a MAT titer $\geq 1: 20$ against at least one serovar. Therefore, the highest titer obtained was used to identify the infecting serogroup. A total of $138(35.66 \%)$ sera samples were tested positive and seven leptospiral serogroups were identified (Table 2). The major circulating leptospiral serogroups was Javanica (112/138, 81.16\%), followed by Pomona (12/138, $8.70 \%)$, Hebdomadis (5/138, 3.62\%), Australis (3/138, 2.17\%), Autumnalis (3/138, 2.17\%), Icterohaemorrhagiae (2/138, 1.45\%) and Grippotyphosa (1/138, 
0.72\%), with positive agglutination titers varying between 20 and 2560 (Table 2). The highest titer detected was 1:2,560 for serogroup Javanica and Pomona, followed by the titer of 1:1,280 for Icterohaemorrhagiae.

Table 2

\Seroprevalence of pathogenic Leptospiral serogroups among rodents samples by microscopic agglutination tes

\begin{tabular}{|c|c|c|c|c|c|c|c|c|c|c|}
\hline $\begin{array}{l}\text { Collected } \\
\text { Locations }\end{array}$ & Numbers & Positive(n) & Prevalence(\%) & Icterohaemorrhagiae & Javanica & Autumnalis & Australis & Pomona & Hebdomadis & Grippc \\
\hline Changtai & 47 & 8 & 17.02 & 0 & 3 & 1 & 0 & 4 & 0 & 0 \\
\hline Wuping & 180 & 79 & 43.89 & 0 & 66 & 2 & 2 & 6 & 2 & 1 \\
\hline Fuqing & 26 & 14 & 53.85 & 0 & 12 & 0 & 0 & 2 & 0 & 0 \\
\hline Luoyuan & 67 & 29 & 43.28 & 0 & 26 & 0 & 0 & 0 & 3 & 0 \\
\hline Jianyang & 36 & 4 & 11.11 & 0 & 3 & 0 & 1 & 0 & 0 & 0 \\
\hline Pucheng & 31 & 4 & 12.9 & 2 & 2 & 0 & 0 & 0 & 0 & 0 \\
\hline Total & 387 & 138 & 35.66 & 2 & 112 & 3 & 3 & 12 & 5 & 1 \\
\hline
\end{tabular}

Seroprevalence was significantly different across the six regions, with the highest prevalence in Fuqing (14/26, 53.85\%), followed by Wuping (79/180, 43.89\%), Luoyuan (29/67, 43.28\%), Changtai (8/47, 17.02), Pucheng (4/31, 12.9\%), and Jianyang (4/36, 11.11\%) (P< 0.001) (Table S8). Serogroups Javanica was the major prevalent serogroup in Wuping, Fuqing, Luoyuan, Jianyang and Pucheng, while Pomona was in Changtai (Table 2).

Seroprevalence was significantly different across species of rodents. The highest MAT seroprevalence corresponded to: $R$. rattoides (98/214, $45.79 \%), R$. flavipectus (18/60, 30.00\%); A. agrarius (2/8, 25.0\%), B. indica $(1 / 4,25.0 \%)(\mathrm{P}<0.05)($ Table S9).

\section{Discussion}

Leptospirosis is a zoonotic disease that is caused by pathogenic Leptospira spp. and is considered endemic in Fujian Province. The role of rodents as a source of Leptospira infection has been suggested worldwide $(1,10)$. However, systematic studies on the primary vector, the rat, and the serological and molecular characteristics of Leptospira circulating among the rat population in Fujian were limited. In this study, we provided the systematic distribution of rodents, the carrier status of Leptospira spp. in rats by culturing and serological detection, molecular characteristics of pathogenic Leptospira circulating in potential reservoirs of rodent populations in Fujian Province during 2018-2020, which plays an important role in the epidemiology of leptospirosis.

Four serogroups of Javanica, Icterohaemorrhagiae, Pyrogenes and Bataviae were identified in these 14 strains isolated from rodents in Fujian, with serogroup Javanica being the most prevalent (50.00\%). In addition, the serological analysis showed that the seroprevalence was $35.66 \%$ and seven serogroups of Javanica, Pomona, Hebdomadis, Australis, Autumnalis, Icterohaemorrhagiae, and Grippotyphosa were found in sera samples in rodent populations by MAT, with Javanica be the major circulating leptospiral serogroup (81.16\%). Therefore, Javanica was the major prevalence serogroup in Fujian, the same prevalence serogroup circulating in Jiangxi reported in our previous studies (9). It was reported that three dominant serogroups named Icterohaemorrhagiae, Javanica, and Australis, were responsible for leptospirosis in Jiangxi Province between 2002 and 2014 (9). The lower diversity of serogroups / STs of leptospirosis in Fujian may be due to a low number of isolates obtained from the rat population. In this study, two most abundant species, $L$. interrogan, and $L$. borgpetersenii, were identified in Fujian, which is consistent with a previous epidemiological investigation of leptospirosis in humans and potentially small animal reservoirs in Jiangxi and worldwide (9). It was reported that four species of $L$. borgpetersenii, L. interrogans, $L$. kirschneri and $L$. weilli were detected in rodents and human isolates in Thailand, Lao PDR, and Cambodia, with L. interrogans and L. borgpetersenii widely distributed amongst rodent populations (11). It was reported that $L$. interrogans, $L$. kirschneri and $L$. borgpetersenii were the most widespread and prevalent species detected in Russia (12). Together, these data revealed that the major Leptospira species from the neighboring countries were similar to each other.

In this study, a total of 6 different STs were obtained from 14 pathogenic Leptospira isolates in Fujian Province, with ST143, ST1, and ST17 being the most prevalent STs (S1 Table). It was reported that the five most predominant STs ( ST1, ST143, ST105, ST37 and ST17) were identified as long-term and ubiquitous virulent strains throughout Jiangxi Province (9). ST143, as the most prevalent STs in Fujian and Jiangxi, was also identified in Malaysia (13). ST17 was also identified in 90 strains of serogroup Icterohaemorrhagiae in Sao Paulo (14). Five common STs (ST37, ST17, ST 199, ST110, and ST146) were reported as a longtime and ubiquitous distribution in Russia (12). Therefore, these prevalent STs (ST17 and ST143) reported in Fujian and Jiangxi in China were also the same prevalent STs in the rest of the world. These prevalent isolates are likely to have adaptive selective advantages in the environment or potential reservoirs, allowing them to circulate worldwide.

In this study, it was revealed the first description of rat species and the density of field rodents was between $4.81-16.77 \%$ in different sampling sites in Fujian Province, which is the similar to the rate in Jiangxi Province in China (4.76-12.56\%) conducted by our previous surveys (9). However, the most abundantly trapped species were different in these two provinces, with $R$. rattoides, $N$. fulvescens, and $R$. flavipectus in Fujian, and $A$. agrarius and $R$. rattoides in Jiangxi. The overall carriage rate $(14 / 710,1.97 \%)$ in rats detected in this investigation based on culture isolation in Fujian were much lower than previous studies in other countries such as Malaysia (6.7\%) (15), Barbados(16-19\%) (16), Brazil (80.3\%) (17). It is important to note that different methods were performed for trapping and determining the presence of leptospires in other labs. The carriage rate of the 6 different species of rodents in Fujian was between $0.71-33.33 \%$. The highest carriage rates based on culture isolation were in $N$. coning $(1 / 3,33.33 \%)$ and $A$. agrarius $(2 / 11,18.18 \%)$ in Fujian, which is consistent with a previous study that $A$. agrarius was the major small animal reservoir in Jiangxi and Guizhou Provinces in China $(9,18) \rrbracket$ 
The infection rates of Leptospira in rodents are varied in different areas around the world. In this study, the seroprevalence of pathogen leptospiral renal carriage in trapped mice using MAT was $35.66 \%$. It was reported that $8 \%$ of the 266 rodents were seropositive, primarily to the serovars Australis and Grippotyphosa in southern Germany (19). It was reported that $27.1 \%$ of $R$. norvegicus were seropositive, and $70 \%$ of the positive serum samples reacted with serogroup Bataviae in northern Vietnam, Hanoi, and Bac Giang (20). It was reported that $68.1 \%$ of the 142 captured $R$. norvegicus were seropositive by MAT titer of $\geq 1: 100$ in Brazil (17). The seroprevalence in rats was 7.1\% in Andaman islands (21). Here, our study found that rodents were heavily infected with Leptospira in Fujian,, indicating a potential risk of rat-borne leptospirosis in this region. Therefore, increased self-protection awareness, and protective measures are needed to prevent Leptospira infections in Fujian.

In this study, the leptospiral diversity of species/serogroups/STs was significantly different across the six other regions. The leptospiral diversity may be due to the difference in special geographic areas. In this study, Jianyang and Pucheng are located in the northern mountain region, Wuping was in the western hilly plain area, and Luoyuan, Fuqing, and Changtai were southeast coastal regions. The differences in altitude, geomorphology, and climate among these areas may influence leptospiral diversity.

This study revealed the prevalence rate and genetically diverse pathogenic Leptospira circulating among the rat populations in Fujian Province, China, suggesting that rodents could be an important Leptospira source. Based on the high infection rate present in this population, these findings highlight the risk of the proximity of the infection reservoirs to humans. Therefore, active and long-term monitoring of infections in wild rodents is necessary, and this may be useful in developing guidelines for the prevention and control of leptospirosis in Fujian Province.

\section{Conclusions}

This study detailed systematic epidemiological investigation, molecular characteristics and seroprevalence of pathogenic Leptospira circulating in potential reservoirs of rodent populations in Fujian Province during 2018-2020. Two pathogenic species, four serogroups and 6 STs were identified in these 14 isolates by $16 S$ rRNA sequencing typing, MAT, and MLST analysis. Significant geographic variations in the distribution of dominant serogroups, species and STs were found among the Leptospira isolates in Fujian. The serological analysis revealed the high seroprevalence rate of leptospirosis among rodent populations in Fujian, indicating a potential risk of rat-borne leptospirosis in this region. Thus, our present study provides a blueprint for further disease prevention and control.

\section{Methods}

\section{Rodents collection and Leptospiral isolation in Fujian Province}

Six different trapping sites representing different geographical locations, including Jianyang (27.33 N; 118.12 E) and Pucheng (27.92 N; 118.53 E) (North), Luoyuan (26.48 N; 119.55 E) and Fuqing (25.42 N; 119.23 E) (East), Wuping (25.10 N; $116.10 \mathrm{E}$ ) (West), Changtai (24.62 N; $117.75 \mathrm{E})$ (South) were selected based on the disease incidence over the past few years in Fujian Province.

Rodents were trapped using the Trap-night method from April to October, 2018 through 2020 within the framework of the CERoPath project (www.ceropath.org) (22). Trap placement was based on indicators of rodent activity such as rice field environments, open sewers, tracks, or feces. Within each sample site, rodents were trapped over an area of approximately 10 kilometers squared. Trapping was conducted with peanut bait on the first afternoon and collected in the following early morning during a period of five days and four nights. For each trapped site, 10 trapping lines, including 10 locally hand-made wire traps (approximately $40 \times 12 \times 12 \mathrm{~cm}$ ) were placed, with at least five meters spacing between traps (22). First, the trapped rodents were identified by gender, genus and species, based on phenotypic characteristics (body, ears, tail, fur color and sex) (23). The rat density was calculated using the formula: (Number of rats trapped each region / Number of total traps successfully placed for each region * 100).

Approximately $1 \mathrm{~g}$ of fresh kidney tissue samples from rats were aseptically inoculated to $10 \mathrm{ml}$ of liquid Ellinghausen-McCullough-Johnson-Harris (EMJH) medium (Difco Laboratories, USA) with 5-fluorouracil (Merck, Germany) at $28^{\circ} \mathrm{C}$ and observed weekly using dark-field microscopy for up to two months.

Species identification of pathogenic Leptospira isolated from Fujian Province

Species identification of the isolates was conducted based on $16 S$ rRNA gene sequencing as previously described by Morey (24). A total of 20 reference sequences representing pathogenic, intermediate and saprophytic Leptospira species were obtained from the GenBank database. Turneriella parva NCTC $11395 \mathrm{~T}$ and Leptonema illini NCTC $11301 \mathrm{~T}$ were set as the outgroup (S2 Table) $(24,25)$. The sequences of all the 14 leptospiral strains from Fujian were compared with the 20 accessible reference sequences using Clustal W. A neighbor-joining tree was constructed using Mega software version 5.10 with a bootstrap value of 1,000 .

\section{Serogroup identification of leptospiral strains isolated from Fujian Province}

For serogroup identification, microscopic agglutination test (MAT) was performed by a panel of 15 Chinese prevalent serogroup-specific rabbit antisera provided by the National Institutes of Food and Drug Control, China. The serogroup scoring the highest MAT titer was defined as the corresponding serogroup. Multilocus sequence typing (MLST) of isolated leptospiral strains

MLST analysis was conducted using seven house-keeping genes ( $g / m U, p n t A$, sucA, tpiA, pfkB, mreA and caiB) as previously described (26). The available PubMLST Leptospira database (http://pubmlst.org/leptospira/) was used for assigning alleles and sequence types (STs). Phylogenetic analysis was conducted using the unweighted pair group method with average linkages provided in BioNumerics software version 5.10. 
Serological antibody detection in rodents using MAT.

Serum samples from the trapped rodents were collected, and MAT analyses were performed for Leptospira-specific antibodies with a panel of 10 local pathogenic Leptospira strains belonging to 10 serogroups (Icterohaemorrhagiae, Javanica, Autumnalis, Australis, Pomona, Hebdomadis, Grippotyphosa, Pyrogenes, Bataviae and Sejroe) as previously described (27). Sera were considered positive for seroreactivity at a MAT titer $\geq 1: 20$ against at least one serovar. The screening was performed with a starting dilution of 1: 10 and up to a dilution of 1:2, 560. MAT end-point titer was defined as the highest dilution at which showing $\geq 50 \%$ agglutination in free-moving leptospires (28).

\section{Statistical analysis}

Fisher's exact test was performed to compare the differences in the distribution of predominate Leptospira across species, serogroups and STs between six different sites. The statistical Kruskal-Wallis Chi-Square, $x^{2}$ tests were used to determine if there were differences in Leptospira positivity rates between other sites and rodent species. The p-value was computed by Monte Carlo simulation. All statistical analyses were conducted using R software (R version 3.5.1, https://www.r-project.org/), considering a significance level of 0.05 .

\section{Abbreviations}

\section{MLST}

Multilocus sequence typing

MAT

microscopic agglutination test

\section{Declarations}

\section{Acknowledgements}

Not applicable.

\section{Authors' contributions}

CZ and GX conceived and designed the experiments; GX, HQ, WL and JW performed the experiments; CZ, GX, XJ and ZL analyzed the data; CZ, FX, YFC and YZ wrote the paper. All authors read and approved the final manuscript.

\section{Funding}

This work was supported by the National Natural Science Foundation of China (No. 81601812), the Medical Innovation Funding of Fujian Province (No. 2019CXB-13), Fujian provincial health technology project (No. 2019-ZQN-27) and Construction of Fujian Provincial Scientific and Technological Innovation Platform (No. 2019Y2001).

\section{Availability of data and materials}

The data generated and/or analyzed during the current study are available from the corresponding author on reasonable request.

\section{Ethics approval and consent to participate}

This study and research protocols were approved by the Ethical Committee of Fujian Center for Disease Control and Prevention, China (No: FJCDCNT18112015). The trapping, handling, and euthanasia of wild rodents in this study were conducted following the protocols approved by the Ethical Committee of Fujian Center for Disease Control and Prevention, China.

\section{Consent for publication}

Not applicable.

\section{Competing interests}

The authors declare that they have no competing interests.

\section{Author details}

${ }^{1}$ Fujian Provincial Key Laboratory of Zoonosis Research, Fujian Center for Disease Control and Prevention, Fuzhou, China. ${ }^{2}$ College of Public Health, Fujian Medical University, Fuzhou, China. ${ }^{3}$ State Key Laboratory for Infectious Disease Prevention and Control, National Institute for Communicable Disease Control and Prevention, Chinese Center for Disease Control and Prevention, Beijing, China. ${ }^{4}$ Collaborative Innovation Center for Diagnosis and Treatment of Infectious Diseases, Hangzhou, China. ${ }^{5}$ Department of Population Medicine and Diagnostic Sciences, College of Veterinary Medicine, Cornell University, Ithaca, New York, USA. ${ }^{6}$ Department of Microbiology and Immunology, Institute of Medical Science, Shanghai Jiao Tong University School of Medicine, Shanghai, China

\section{References}


1. Levett PN. Leptospirosis. Clinical microbiology reviews. 2001;14(2):296-326.

2. Adler B. History of leptospirosis and leptospira. Curr Top Microbiol Immunol. 2015;387:1-9.

3. Costa F, Hagan JE, Calcagno J, Kane M, Torgerson P, Martinez-Silveira MS, et al. Global Morbidity and Mortality of Leptospirosis: A Systematic Review. PLoS Negl Trop Dis. 2015;9(9):e0003898.

4. Guglielmini J, Bourhy P, Schiettekatte O, Zinini F, Brisse S, Picardeau M. Genus-wide Leptospira core genome multilocus sequence typing for strain taxonomy and global surveillance. PLoS Negl Trop Dis. 2019;13(4):e0007374.

5. Picardeau M. Virulence of the zoonotic agent of leptospirosis: still terra incognita? Nat Rev Microbiol. 2017;15(5):297-307.

6. Victoriano AF, Smythe LD, Gloriani-Barzaga N, Cavinta LL, Kasai T, Limpakarnjanarat K, et al. Leptospirosis in the Asia Pacific region. BMC infectious diseases. 2009;9:147.

7. Ellis WA. Animal leptospirosis. Curr Top Microbiol Immunol. 2015;387:99-137.

8. Plank R, Dean D. Overview of the epidemiology, microbiology, and pathogenesis of Leptospira spp. in humans. Microbes and infection / Institut Pasteur. 2000;2(10):1265-76.

9. Zhang C, Xu J, Zhang T, Qiu H, Li Z, Zhang E, et al. Genetic characteristics of pathogenic Leptospira in wild small animals and livestock in Jiangxi Province, China, 2002-2015. PLoS Negl Trop Dis. 2019;13(6):e0007513.

10. Adler B, de la Pena Moctezuma A. Leptospira and leptospirosis. Vet Microbiol. 2010;140(3-4):287-96.

11. Cosson JF, Picardeau M, Mielcarek M, Tatard C, Chaval Y, Suputtamongkol Y, et al. Epidemiology of leptospira transmitted by rodents in southeast Asia. PLoS Negl Trop Dis. 2014;8(6):e2902.

12. Voronina OL, Kunda MS, Aksenova El, Ryzhova NN, Semenov AN, Petrov EM, et al. The characteristics of ubiquitous and unique Leptospira strains from the collection of Russian centre for leptospirosis. BioMed research international. 2014;2014:649034.

13. Benacer D, Mohd Zain SN, Ahmed AA, Mohd Khalid MKN, Hartskeerl RA, Thong KL. Predominance of the ST143 and ST50 Leptospira clones in the urban rat populations of Peninsular Malaysia. J Med Microbiol. 2016;65(6):574-7.

14. Romero EC, Blanco RM, Galloway RL. Analysis of multilocus sequence typing for identification of Leptospira isolates in Brazil. Journal of clinical microbiology. 2011;49(11):3940-2.

15. Benacer D, Mohd Zain SN, Amran F, Galloway RL, Thong KL. Isolation and molecular characterization of Leptospira interrogans and Leptospira borgpetersenii isolates from the urban rat populations of Kuala Lumpur, Malaysia. Am J Trop Med Hyg. 2013;88(4):704-9.

16. Levett PN, Walton D, Waterman LD, Whittington CU, Mathison GE, Everard CO. Surveillance of leptospiral carriage by feral rats in Barbados. West Indian Med J. 1998;47(1):15-7.

17. de Faria MT, Calderwood MS, Athanazio DA, McBride AJ, Hartskeerl RA, Pereira MM, et al. Carriage of Leptospira interrogans among domestic rats from an urban setting highly endemic for leptospirosis in Brazil. Acta Trop. 2008;108(1):1-5.

18. Li S, Wang D, Zhang C, Wei X, Tian K, Li X, et al. Source tracking of human leptospirosis: serotyping and genotyping of Leptospira isolated from rodents in the epidemic area of Guizhou province, China. BMC Microbiol. 2013;13:75.

19. Mayer-Scholl A, Hammerl JA, Schmidt S, Ulrich RG, Pfeffer M, Woll D, et al. Leptospira spp. in rodents and shrews in Germany. Int J Environ Res Public Health. 2014;11(8):7562-74.

20. Koizumi N, Miura K, Sanai Y, Takemura T, Ung TTH, Le TT, et al. Molecular epidemiology of Leptospira interrogans in Rattus norvegicus in Hanoi, Vietnam. Acta Trop. 2019;194:204-8.

21. Sharma S, Vijayachari P, Sugunan AP, Sehgal SC. Leptospiral carrier state and seroprevalence among animal population-a cross-sectional sample survey in Andaman and Nicobar Islands. Epidemiol Infect. 2003;131(2):985-9.

22. Morand. S, Bordes. F, Blasdel. K, Pilosof. S, Cornu. JF, Chaisiri. K, et al. Assessing the distribution of disease-bearing rodents in human-modified tropical landscapes. Journal of Applied Ecology. 2015;52(3):784-94.

23. Pages M, Chaval Y, Herbreteau V, Waengsothorn S, Cosson JF, Hugot JP, et al. Revisiting the taxonomy of the Rattini tribe: a phylogeny-based delimitation of species boundaries. BMC Evol Biol. 2010;10:184.

24. Morey RE, Galloway RL, Bragg SL, Steigerwalt AG, Mayer LW, Levett PN. Species-specific identification of Leptospiraceae by $16 \mathrm{~S}$ rRNA gene sequencing. Journal of clinical microbiology. 2006;44(10):3510-6.

25. Smythe L, Adler B, Hartskeerl RA, Galloway RL, Turenne CY, Levett PN. Classification of Leptospira genomospecies 1, 3, 4 and 5 as Leptospira alstonii sp. nov., Leptospira vanthielii sp. nov., Leptospira terpstrae sp. nov. and Leptospira yanagawae sp. nov., respectively. International journal of systematic and evolutionary microbiology. 2013;63(Pt 5):1859-62.

26. Boonsilp S, Thaipadungpanit J, Amornchai P, Wuthiekanun V, Bailey MS, Holden MT, et al. A single multilocus sequence typing (MLST) scheme for seven pathogenic Leptospira species. PLoS neglected tropical diseases. 2013;7(1):e1954.

27. Marquez A, Ulivieri T, Benoit E, Kodjo A, Lattard V. House Mice as a Real Sanitary Threat of Human and Animal Leptospirosis: Proposal for Integrated Management. Biomed Res Int. 2019;2019:3794876.

28. Ngugi JN, Fevre EM, Mgode GF, Obonyo M, Mhamphi GG, Otieno CA, et al. Seroprevalence and associated risk factors of leptospirosis in slaughter pigs; a neglected public health risk, western Kenya. BMC Vet Res. 2019;15(1):403.

\section{Figures}




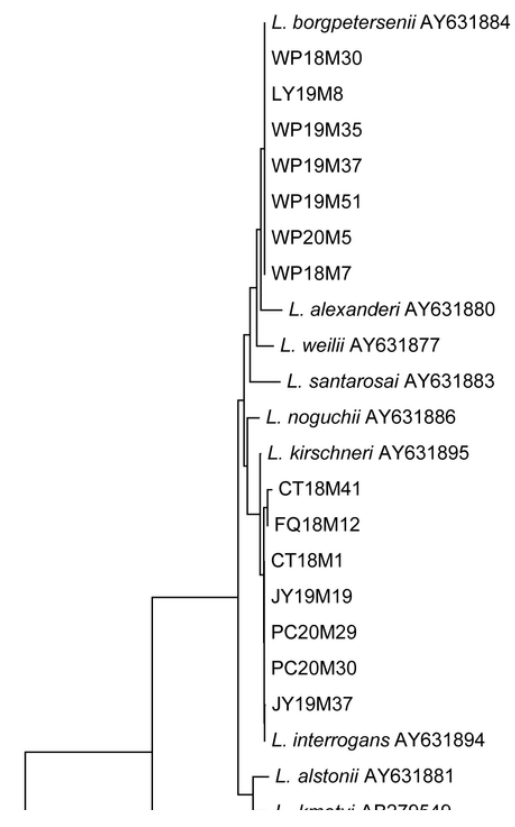

\section{Figure 1}

Phylogenetic analysis based on 16SrRNA gene for the 14 pathogenic Leptospira strains in Fujian.

Two species of L. interrogans and L. borgpetersenii were identified among these 14 Leptospira strains. 
Categorical

MLST

\begin{tabular}{|c|c|c|c|}
\hline 유 & Strain & Year & Source \\
\hline \multirow[t]{4}{*}{ Clade 1} & PC20M29 & 2020 & Apodemus agrarius \\
\hline & РC20M30 & 2020 & Apodemus agrarius \\
\hline & JY19M19 & 2019 & Niviventer fulvescens \\
\hline & JY19M37 & 2019 & Niviventer niviventer \\
\hline
\end{tabular}

$\begin{array}{lccc}\text { Species } & \text { Serogroup } & \text { ST } & \text { region } \\ \text { L. interrogans } & \text { Icterohaemorrhagiae } & 1 & \text { pucheng } \\ \text { L. interrogans } & \text { Icterohaemorrhagiae } & 1 & \text { pucheng } \\ \text { L. interrogans } & \text { Icterohaemorrhagiae } & 17 & \text { jianyang } \\ \text { L. interrogans } & \text { Icterohaemorrhagiae } & 17 & \text { jianyang }\end{array}$

Figure 2

UPGMA dendrogram indicating the diversity of 14 pathogenic Leptospira strains isolated from Fujian Province in MLST analysis.

A similarity of $60 \%$ defined groups. The dendrogram reveals that the 14 pathogenic Leptospira strains belonged to five major clades.

\section{Supplementary Files}

This is a list of supplementary files associated with this preprint. Click to download.

- Additionalfile1.xIsx

- Additionalfile2.doc

- Additionalfile39.xlsx 